\title{
A new tool for the action potential repolarization dynamic analysis: Application to the discrimination of diabetic and control cells
}

\author{
O. Meste ${ }^{1}$, M. $\mathrm{Meo}^{2}$, S. Signore ${ }^{3}$, M. Rota ${ }^{4}$ \\ ${ }^{1}$ Université Côte d'Azur, CNRS, I3S, France \\ ${ }^{2}$ IHU Liryc, Electrophysiology and Heart Modeling Institute, fondation Bordeaux Université, France \\ ${ }^{3}$ Department of Anesthesia, Brigham and Women's Hospital, Boston, MA, US \\ ${ }^{4}$ Department of Physiology, New York Medical College, Valhalla, NY, US
}

\begin{abstract}
In CinC2015 we have shown that the dynamics of the action potential (AP) repolarization could be tracked throughout the stimulation course. Despite some valuable outcomes, the populations of interest (control, Ctrl, and streptozotocin-induced, STZ, diabetic mice) could not be significantly distinguished in term of dynamics because of the global extracted feature. In this study, the computation of new features for each repolarization percentage allows an accurate and meaningful characterization of the two groups leading to a significant classification. APs in isolated left ventricular cardiomyocytes obtained from Ctrl and STZ mice were measured by patch-clamp. The progressive changes in AP repolarization for individual cells were tested on a set of 100 consecutive excitations at $2 \mathrm{~Hz}$ pacing rate. The corresponding repolarizations are stacked in a matrix decomposed with a new approach. Observations are modeled as a sum of vectors multiplied by specific polynomial functions. This approach is similar to the Singular Value Decomposition (SVD), but the corresponding scalars are replaced by these functions. Model unknowns are estimated by using an alternated least square algorithm. Finally, the mean of the polynomial first derivative is computed for each repolarization percentage as a representative feature. A Wilcoxon signed rank test $(p<0.05)$ has been applied on the features from the two groups. We can observe a significant difference in the late repolarization phase (70\%-95\% repolarization), with a singular behavior in correspondence with the AP profile shoulder onset (80\%).
\end{abstract}

\section{Introduction}

The duration of the action potential (AP) in cardiomyocytes is an important variable controlling the electrical properties of the normal and pathological myocardium. Prolongation of the AP in myocytes may represent the ba- sis for the increased risk of arrhythmia with diabetes [1], but the determinants of these abnormalities remain to be elucidated. We have shown in [2] that the diabetic condition is associated with alterations in the temporal dynamics of the AP profile in myocytes, a factor that may originate electrical instability. This assessment has been performed by using statistics computed over APs associated to isolated left ventricular myocytes obtained from control (Ctrl)and streptozotocin-induced (STZ) diabetic mice. In [3], these statistical properties (mean and variance) have been replaced by the analysis of the temporal dynamics of the repolarization phase. It has been shown that the two groups could be partly distinguished by introducing a new automatic analysis based on a transformation of each AP.

The objective of this paper is not restricted to only fully distinguish the two groups but also to produce additional predictive information on the ionic currents involved in the observed differences. For instance, if the groups are significantly different at specific percentages of repolarization, it implies that ionic currents active in these membrane potential ranges are affected by the hyperglycemic condition. To achieve this task, we introduced a new decomposition method, well adapted to the observation, based on the aforementioned AP transformation. It is shown that the proposed approach succeeded in distinguishing the electrical behavior of the two groups of cells, corroborating the impact of diabetes on repolarizing Kv currents.

\section{Material}

A group of female $\mathrm{C} 57 \mathrm{Bl} / 6$ mice between 3 and 4.4 months of age was studied in accordance with the Guide for Care and Use of Laboratory Animals; experiments were approved by the local animal care committee (IACUC). Hyperglycemia was induced by injecting animals with streptozotocin (Sigma, concentration $5 \mathrm{mg} / \mathrm{L}$ ) for approximately one week. Blood glucose levels were measured 6-10 days after the last injection of STZ. Mice 
with blood glucose concentration higher than $400 \mathrm{mg} / \mathrm{dl}$ were ascribed to the diabetic group. Furthermore, we examined only animals at less than 1 month after the onset of hyperglycemia, so as to minimize the interference of other factors (e.g., structural or mechanical changes) on myocardial electrical activity.

A set of 41 cells was isolated from left ventricle in 14 control mice, whereas the STZ group consisted of 76 cells obtained from 10 animals. Isolated cardiomyocytes were placed in a bath of Tyrode solution on the stage of an IX53 (Olympus) microscope for whole-cell patch-clamp measurements. APs were assessed in current-clamped myocytes through an Axoclamp 900A amplifier (Molecular Devices). Electrical signals were digitized using a $250 \mathrm{kHz}$ 16-bit resolution A/D converter (Digidata 1550, Molecular Devices) and recorded through pCLAMP 9.010 software (Molecular Devices) with low-pass filtering at $2 \mathrm{kHz}$. Recording pipettes were fabricated using a horizontal ( $\mathrm{P}$ 1000 , Sutter Instrument) glass microelectrode puller. Current pulses 1.5 times threshold were applied for cell stimulation. A sequence of 100 APs has been triggered by the stimulation protocol at a $2 \mathrm{~Hz}$ pacing rate.

\section{Method}

The goal of the presented method is not to measure an absolute trend in the observation, for each beat and for each repolarization magnitude, but to determine how AP profile dynamics is globally affected throughout the stimulation sequence. Indeed, it can be seen as an extension of the singulat value decomposition (SVD) approach where an observation can be represented as the weighted sum of vectors, and each of its weights is not a single scalar value, but a function. For the SVD, the decomposition of each AP observation vector $\mathbf{x}_{i}(i=1, \ldots, I)$ is defined by:

$$
\mathbf{x}_{i}=a_{1, i} \mathbf{v}_{1}+a_{2, i} \mathbf{v}_{2}+a_{1, i} \mathbf{v}_{3}+\ldots
$$

where the vectors $\mathbf{v}_{j}$ are constrained to be orthogonal and normalized. This decomposition does not take into account any temporal structures hidden in vectors $\mathbf{x}_{i}$, meaning that if their components are shuffled the corresponding $\mathbf{v}_{j}$ will be shuffled accordingly and producing the same $a_{j, i}$.

The new model we propose consists in decomposing the observations such that :

$$
\mathbf{x}_{i}=\mathbf{p}_{1, i} \circ \mathbf{v}_{1}+\mathbf{p}_{2, i} \circ \mathbf{v}_{2}+\mathbf{p}_{3, i} \circ \mathbf{v}_{3}+\ldots
$$

The symbol 'o' stands for the Hadamard product corresponding to the element-wise product. The application of this decomposition to the AP matrix consists in considering a $\mathbf{x}_{i}$ as the successive time values throughout the stimulations, for a given amplitude level. In this case we assume a temporal structure captured by both the $\mathbf{p}_{j, i}$ and $\mathbf{v}_{j}$ that will allow a lower complexity of the decomposition compared to (1). When using SVD, the variation information is often shared by different components, thus making its interpretation more difficult. In other words, it is expected that the new modeling would facilitate this task because of the lower number of summation terms. For our practical case, the model will be approximated by an order 1 decomposition:

$$
\mathbf{x}_{i}=\mathbf{p}_{i} \circ \mathbf{v}+\mathbf{e}_{i}
$$

with $\mathbf{e}_{i}$ the approximation error.

Assuming that the vector $\mathbf{p}_{i}$ is fully parameterized by using a linear combination of basis vectors, that is:

$$
p_{i}(n)=\sum_{k=0}^{K-1} b_{k}(n) \theta_{i, k}
$$

with $b_{k}(n)$ a polynomial basis for instance, the minimization of $\sum_{i=1}^{I}\left\|\mathbf{e}_{i}\right\|_{2}^{2}$ provides an estimate of the parameters of $\mathbf{p}_{i}$ and $\mathbf{v}$, with $\|\mathbf{v}\|_{2}^{2}=1$. The latter constraint avoids indeterminacies of the scaling and imposes the uniqueness of the decomposition. This estimation is performed by using an alternated least squares approach with the two following solutions:

$$
\hat{\mathbf{v}}=\left(\sum_{i=1}^{I} \operatorname{diag}\left(\mathbf{p}_{i}\right)^{2}\right)^{-1}\left(\sum_{i=1}^{I}\left(\mathbf{x}_{i} \circ \mathbf{p}_{i}\right)\right)
$$

and

$$
\begin{gathered}
\mathbf{p}_{i}=\mathbf{M}_{\hat{v}} \hat{\boldsymbol{\theta}}_{i} \\
\hat{\boldsymbol{\theta}}_{i}=\left(\mathbf{M}_{\hat{v}}^{T} \mathbf{M}_{\hat{v}}\right)^{-1} \mathbf{M}_{\hat{v}}^{T} \mathbf{x}_{i}
\end{gathered}
$$

with $\mathbf{M}_{\hat{v}}=\mathbf{B} \circ\left(\hat{\mathbf{v}} \mathbf{I}^{T}\right)$ and where the $b_{k}(n)$ are stored column-wise in $\mathbf{B}$ and $\mathbf{I}$ stands for the unit vector.

Note that for the full extension in (2), by using similar approach to the SVD computation, all the unknowns can be estimated with the constraints that the summation terms are orthogonal (but not the $\mathbf{v}_{j}$ ) and $\left\|\mathbf{v}_{j}\right\|_{2}^{2}=1$.

Once the $\mathbf{p}_{i}$ are estimated the mean $\mathbf{p}$ is computed as $\overline{\mathbf{p}}=\frac{1}{I} \sum_{i} \mathbf{p}_{i}$ and $\tilde{\mathbf{v}}=\overline{\mathbf{p}} \circ \mathbf{v}$ such that $\frac{1}{I} \sum_{i} \mathbf{x}_{i}=\tilde{\mathbf{v}}$, neglecting the approximation error. This also allows to write $\mathbf{x}_{i}=\tilde{\mathbf{p}}_{i} \circ \tilde{\mathbf{v}}$ with $\tilde{p}_{i}(n)=p_{i}(n) / \bar{p}_{i}(n)$. This normalization step is crucial because the algorithm could converge toward $-\mathbf{p}_{i}$ and $-\hat{\mathbf{v}}$ instead of $\mathbf{p}_{i}$ and $\hat{\mathbf{v}}$ while the Hadamard product of the two vectors produces the same result. This is important in order to compare the derivatives of the $\tilde{p}_{i}(n)$ for different observation sets regardless this indeterminacy.

In our application each $\mathbf{p}_{i}$ is modeled as an order 3 polynomial function with parameters to be estimated. The originality of the approach is to not assess the discriminative power of the $\tilde{\mathbf{v}}$, however distinct for each cell, but to focus on the property of the transformed polynoms $\tilde{\mathbf{p}}_{i}$. If for a given AP magnitude the polynom tends to decrease the trend of the time values throughout the time, then it should 
be observed through the mean of its derivative, regardless the profile of the corresponding $\tilde{\mathbf{v}}$. The main property of $\tilde{\mathbf{v}}$ is to summarize all the common information shared by all the $\mathbf{x}_{i}$ in a different manner than with the SVD. For a given cell and for each $i$ value, according to each AP magnitude, the mean of the derivative of $\tilde{\mathbf{p}}_{i}$ is computed and stored. The discrimination of the diabetic and the control cells will be based on these last values.

It has been shown in [2] that, according to the mean AP for the two groups, it is more relevant to normalize the observations to avoid bias for the groups discrimination. To achieve this task, the previously computed parameters are normalized accordingly.

\section{Results}

The idea is to distinguish the control (41 records) from the diabetic (76 records) myocells specifically on the repolarization phase. First, for each AP a transformation is applied in order to get a strictly decreasing curve, which in turn is also transformed by computing its inverse [3]. Then for each stimulation sequence (the 30 first have been discarded) we get a matrix of 70 inverse repolarizations (see a sequence of stimulated control cell in Figure 1 and the corresponding $3 \mathrm{D}$ visualization of the corresponding matrix in Figure 2). Note that the horizontal axis stands for the normalized amplitude and the vertical one for the time. The application of the proposed decomposition to the APs consists in considering a $\mathbf{x}_{i}$ as a column of the matrix.

The derivatives, computed over the stimulation index, of the corresponding $\tilde{\mathbf{p}}_{i}$, are given in Figure 3 and will be used to characterize the trend as previously explained. A ranksum test has been computed over the mean of the derivatives in order to discriminate the two groups and is given in Figure 4. In order to interpret the intervals of significance of this test, the mean AP and its corresponding derivative is plotted for the two groups in Figure 5. Finally, the median value of the discriminative parameter is computed for the two groups in Figure 6.

\section{Discussion and conclusion}

In previous works [2],[3] applied to a subset of the records it has been shown that automatic AP analysis can be performed accurately. It has not been demonstrated that the dynamics of the repolarization phase throughout the stimulation allows to distinguish the two populations while some specificity has been found. This could be due to the inherent random variability of the repolarization regardless the groups. While the classification of the two groups is not really relevant in this context, the related tools could reveal new insights of the diabetes effects over the repolarization. From the example given in Figure 3, it is clear that the multiplicative polynoms $\mathbf{p}_{i}$ convey complex infor-

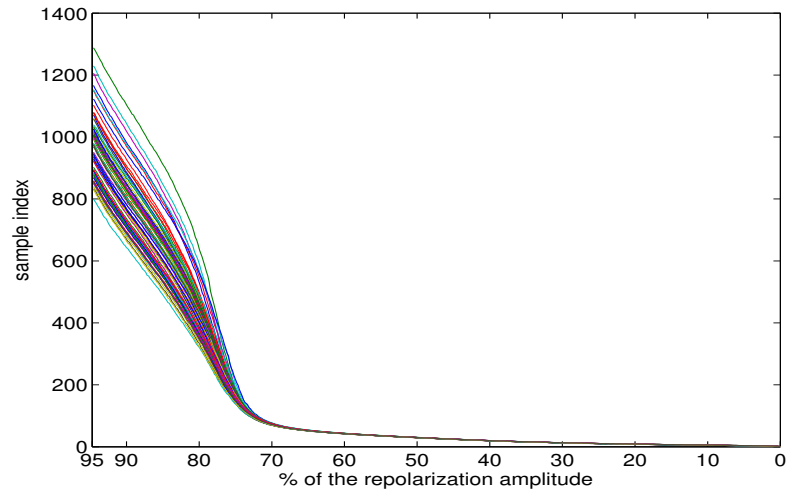

Figure 1. 70 superimposed repolarizations of a control cell

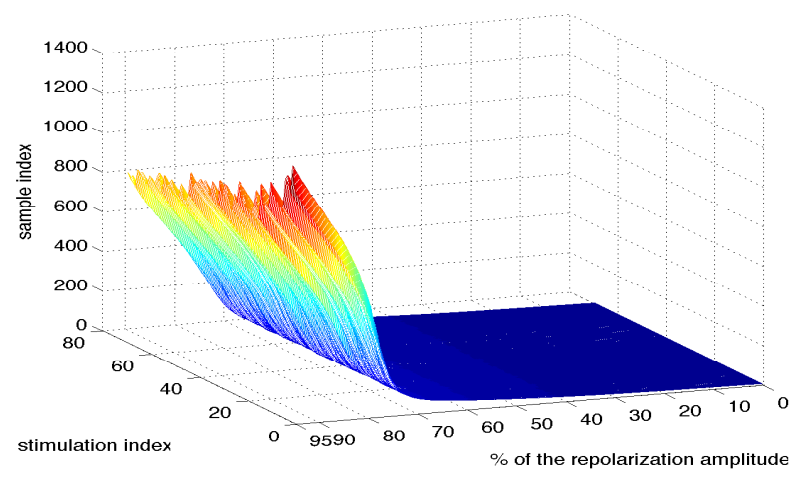

Figure 2. 3D representation of the matrix where the 70 repolarizations have been stacked

mation. The computation of their derivatives succeeded in classifying the two groups, as shown in Figure 4.

It is noticeable that the two groups are significantly different only around the shoulder of the mean AP (see Figure 5 for localization) and mostly in the late repolarization phase. Referring to [4], this localization could be due to changes of the $\mathrm{Kv}$ currents profile. In addition to the work in [2], where it is shown that these changes are due to the effect of diabetes, this new findings could help to identify which Kv currents are the most affected. Furthermore, the median value shown in Figure 6, brings additional information on the dynamics. The diabetic group exhibits a shortening trend (negative values) mostly for the late repolarization phase and conversely for the early phase. For the control group a shortening is observed only in the interval [80-65]\% corresponding to the location of the mentioned shoulder. This result extend the observations given in [5] where similar behaviors are shown to be frequency dependent.

In order to investigate the role of specific Kv currents involved in the observed electrical alterations, new targeted experiments are needed. Alternatively, in silico models 


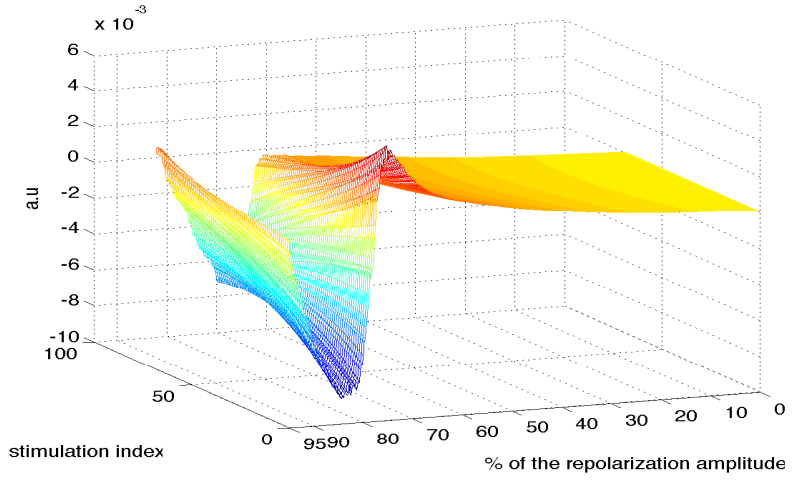

Figure 3. The derivative with respect to stimulation index of the multiplicative estimated polynomials.

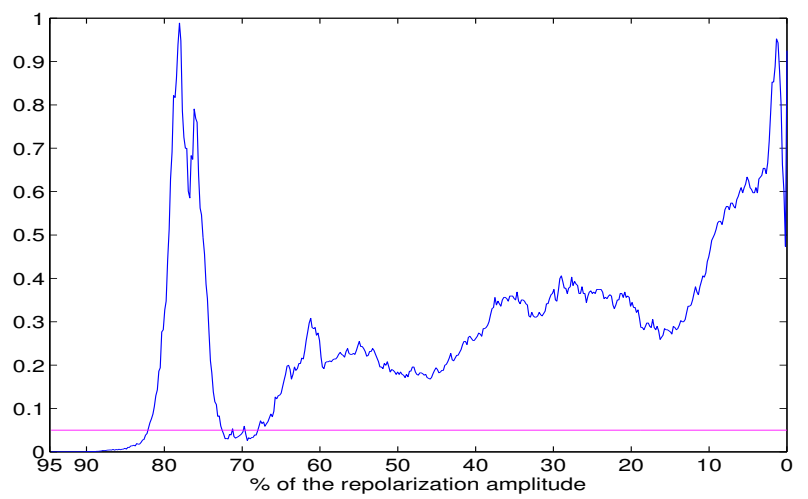

Figure 4. Comparison of the two populations (Ranksum). Significant when $\leq 0.05$.

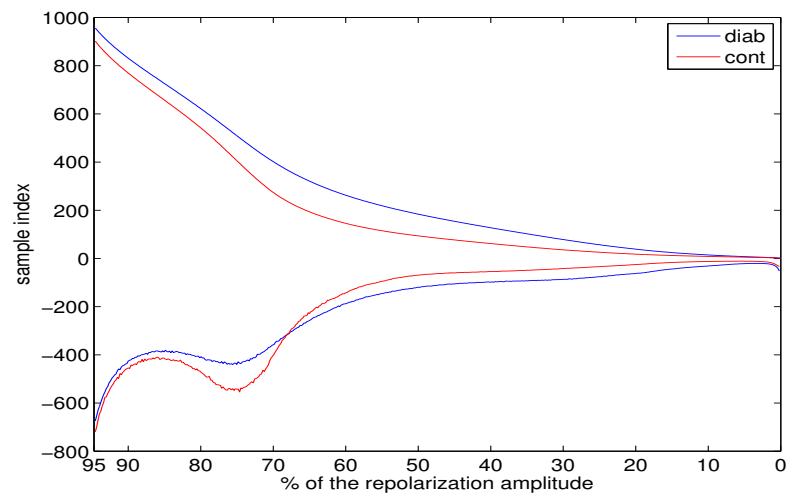

Figure 5. Mean and derivative (magnified by 100) of the control and diabetic populations. Note the shoulder shape in the interval [80-70].

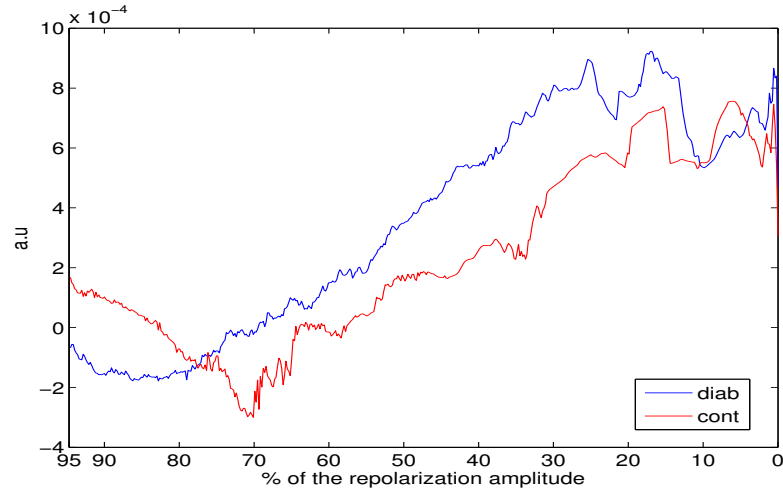

Figure 6. Median value of the discriminant parameter for the two populations

may be combined with our results to identify changes in ionic conductances accounting for the remodeled repolarization of the AP.

\section{References}

[1] Tomaselli GF, Beuklemann DJ, Calkins HG, Berger RD, Kessler PD, Lawrence JH, Kass D, Feldman AM, Marban E. Sudden cardiac death in heart failure: The role of abnormal repolarisation. Circulation. 1994;90:2534-2539.

[2] Meo M, Meste O, Signore S, Sorrentino A, Cannata A et al. Reduction in Kv Current Enhances the Temporal Dispersion of the Action Potential in Diabetic Myocytes: Insights from a Novel Repolarization Algorithm. Journal of the American Heart Association, 5 (2), 2016, 10.1161/JAHA.115.003078.

[3] Meste O, Meo M, Signore S, Rota M. Diabetes affects the temporal dynamics of the repolarization properties of cardiomyocites. Computing in Cardiology, Sep 2015.

[4] Nerbonne J, Kass R. Molecular Physiology of cardiac repolarization. Physiol. Rev., 85: 1205-1253, 2005.

[5] Nobe S, Aomine M, Arita M et al. Chronic diabetes mellitus prolongs action potential duration of rat ventricular muscles: circumstantial evidence for impaired Ca2+ channel. Cardiovasc Research 1990;24(5):381-9.

Address for correspondence:

Pr. Olivier MESTE

Laboratoire I3S - CNRS - UNS

2000 route des Lucioles

06903 Sophia Antipolis cedex, FRANCE

E-mail address: olivier.meste@unice.fr 